\title{
Energy Budgets and Internal Heat of Planets and Moons
}




\section{Radiant Energy Budgets and Internal Heat of Planets and Moons}

L. Li (Univ. of Houston), R. A. West (NASA/JPL), M. E. Kenyon (NASA/JPL), C. A. Nixon (NASA/GSFC), P. M. Fry (UW-Madison), D. Wenkert (NASA/JPL), M. D. Hofstadter (NASA/JPL), X. Jiang (Univ. of Houston),

E. C. Creecy (Univ. of Houston), A. Sanchez-Lavega (UPV/EHU), K. H. Baines (NASA/JPL),

A. Mallama (Univ. of Maryland), R. Hu (NASA/JPL), R. K. Achterberg (Univ. of Maryland), S. Aslam (NASA/GSFC),

D. Banfield (Cornell Univ.), U. Dyudina (SSI), J. J. Fortney (UCSC), A. P. Ingersoll (Caltech), A. Kleinböhl (NASA/JPL),

L. Fletcher (Univ. of Leicester), S. Limaye (UW-Madison), M. S. Marley (NASA Ames), M. D. Smith (NASA/GSFC),

K. M. Soderlund (UT Austin), L. J. Spilker (NASA/JPL), C. L. Young (NASA LaRC)

Abstract: Knowledge of the radiant energy budgets and internal heat of planets and moons is of wide interest in the planetary science community. Some progress has been achieved with recent studies, but there are still significant limitations in current observations and studies. We recommend future exploration to better understand the radiant energy budgets and internal heat of planets and moons in our solar system.

\section{Big picture and significance}

As a fundamental parameter of planets and moons, the radiant energy budget is determined by the absorbed solar energy and the emitted thermal energy $(1,2)$. Such an energy budget plays an important role in determining the thermal structures of planets and moons (3-6). It can help us understand the geology (e.g., polar ices of Mars) (7), internal heat related to the formation and evolution of giant planets [8-10], and sub-surface internal heat driving the jet plumes on some moons (11-15). For bodies with atmospheres, the radiant energy budgets at the top of atmospheres also set critical boundary conditions for the atmospheric systems (3). The transfer and distribution of radiant energies within the atmospheric systems modify the thermal structure to generate available potential energy. The available potential energy can be converted into kinetic energy to drive atmospheric circulation and the related weather and climate $(3-6,16,17)$.

Unfortunately, the global radiant energy budget has not been well determined for most of planets and moons (4-6) in our solar system, mainly because the observations of the radiant energies are limited $(1,2,18,19)$. With the advance of space exploration, we expect to get a much better picture of the global radiant energy budget and internal heat for the planets and moons in our solar system.

\section{Methodology and observations}

To determine the radiant energy budgets and hence the internal heat of planets and moons, we have to measure two radiant energies - the absorbed solar energy and the emitted thermal energy. The emitted thermal energy of planets and moons in our solar system is concentrated in the infrared wavelengths, which can be measured by an instrument in the wavelength range 5-400 microns. On the other hand, the solar energy from the Sun is mainly concentrated in the ultraviolet, visible and near-infrared wavelengths (0-5 microns). Generally, we measure the reflected solar energy and then compute the absorbed solar energy.

The precise measurements of the emitted thermal energy and the reflected solar energy require accurate observations with complete coverage of wavelength and viewing angles (e.g., emission angle and phase angle). The basic methodology of computing the radiant energies is to integrate the radiance over wavelength and viewing angle, which is described in detail in our previous studies $(18,19)$. The difference between the emitted thermal energy and the absorbed solar energy is generally used to estimate the internal heat of planets and moons 
$(1,2)$. To better understand the radiant energy budgets of planets and moons, the spatiotemporal variations of the radiant energies (i.e., the absorbed solar energy and the emitted thermal energy) should be addressed, as shown in our previous studies (18, 19, 20-25).

\section{Recent Progress and challenges}

There has been significant progress in understanding the radiant energy budgets and internal heat of planets and moons from studies by multiple research groups. However, there are significant limitations in current observations and studies. It should be a priority to address those in the next decade.

\subsection{Terrestrial bodies}

\subsubsection{Terrestrial bodies with Earth-like atmospheres (i.e., surface pressure around 1 bar)}

In our solar system, Earth and Titan are two terrestrial bodies that have both significant atmosphere and surface seas. In addition, the surface pressures of the two bodies are comparable (Earth 1 bar and Titan 1.5 bar). For Earth, some recent studies (26-30) revealed a small energy imbalance: the absorbed solar energy exceeds the emitted thermal energy by a magnitude of $0.2-04 \%$ of the emitted energy. These studies further suggest that such a small energy imbalance has a significant impact on the global warming and climate change on Earth.

Titan's global energy budget had not been well determined before the Cassini epoch.

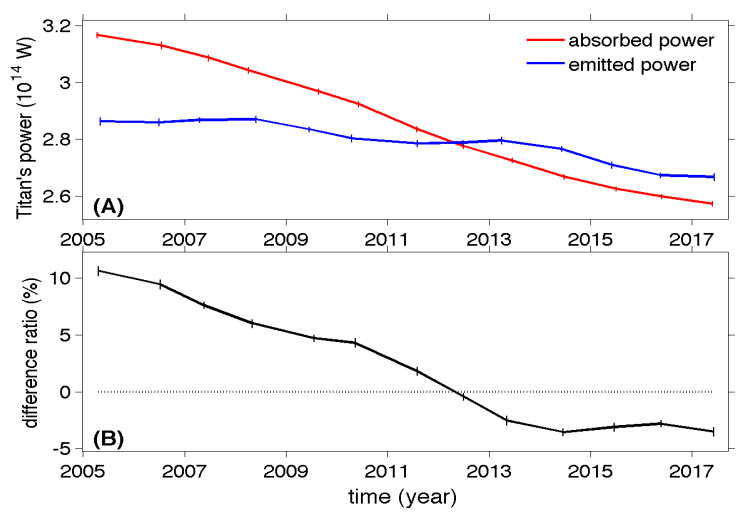

Figure 1. Titan's radiant energies. (A) Comparison between the absorbed solar power and the emitted thermal power. (B) The ratio between the net radiant energy (i.e., absorbed power emitted power) and the emitted power. All current theories and models assume that the global radiant energy budget is balanced (4-6, 31-35). However, the Cassini observations suggest that Titan's radiant energy budget is not balanced (24). The energy imbalance can be beyond $10 \%$ of the emitted energy on a time scale of one Earth year (Fig. 1). Averaging over the Cassini era (2004-2017), the energy imbalance is $2.73 \pm 0.46 \%$ of the emitted energy. Extending the analyses from the Cassini era to a complete Titan year ( 29.4 Earth years), the energy imbalance is $4.78 \pm 0.91 \%$ of the emitted energy (24).

Titan's energy imbalance is much larger than Earth's energy imbalance $(\sim 0.2-0.4 \%$ of the emitted energy), so we expect Titan's energy imbalance has important impacts on its climate system. The current theories and models of Titan's atmosphere and climate, in which the global radiant energy balance is assumed, should be re-examined. In addition, the roles of the energy imbalance in the atmospheric and climate systems of Titan should be examined with the greenhouse and anti-greenhouse effects (36). Furthermore, the energy imbalances on Earth and Titan also suggest that there are probably more terrestrial planets and moons having unbalanced radiation budgets. Unfortunately, the global energy budgets for most of these terrestrial bodies in our solar system have not been well determined. The more we understand the factors influencing 'natural' energy balance on planets and moons, the better we can understand climate change on Earth (e.g., anthropogenic vs non-anthropogenic factors). 


\subsubsection{Terrestrial bodies without global atmospheres}

There are many terrestrial bodies without global atmospheres, which include the planet Mercury and many moons (e.g., Earth's moon and most of giant planets' moons).

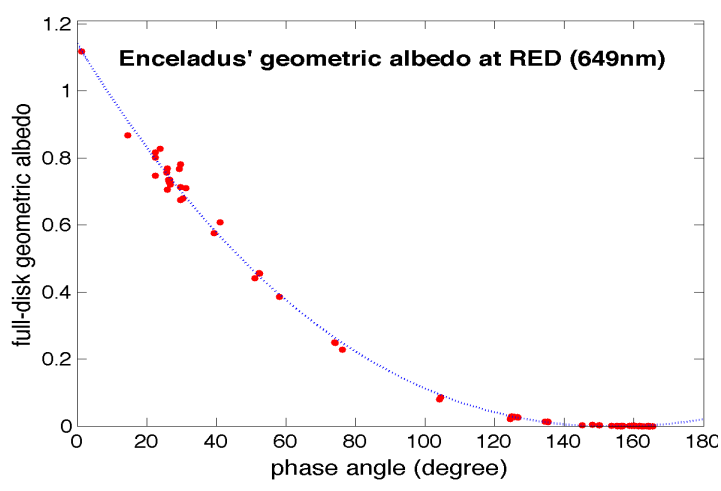

Figure 2. Full-disk albedo of Enceladus recorded by the Cassini observations.
Among them, Enceladus is attracting more and more attention because of its south polar jets. Enceladus' radiant energy budget in the polar regions has been examined by the Cassini and HST observations (11-14), but studies of the global radiant energy budget are still lacking. Based on observations from the Cassini spacecraft, we have conducted preliminary measurements of Enceladus' full-disk albedo (Fig. 2).

Measuring the emitted thermal power from Enceladus is relatively difficult because its surface temperature has large diurnal variations and the temperature is significantly affected by the incident solar radiance. In addition, the heterogeneous surface makes the global measurements of Enceladus' emitted power even harder. We are still working on the measurements of Enceladus' global emitted power based on Cassini observations. It should be mentioned that studies of Enceladus' global radiation budget and hence the internal heat shed light on the putative jets on Europa, which is planned to be visited by Europa Clipper.

\subsubsection{Terrestrial bodies with thin atmospheres.}

There are some terrestrial bodies with thin atmospheres in our solar system (e.g., Mars, Pluto, and Triton).

Mars has been visited by many spacecraft and landers. Regional energy budgets were addressed $(37,38)$. But Mars' global radiant energy budget has not been well determined yet, even though there are some preliminary estimates (4-6, 39). The Thermal emission spectrometer (TES) on the Mars Global Surveyor recorded long-term observations (40), which provide an opportunity to examine Mars' global radiant energy budget and its temporal variations. The observations recorded by the TES broadband thermal emission sensor (Fig. 3) can be used to compute Mars' emitted power. In principle, the observations recorded by the TES

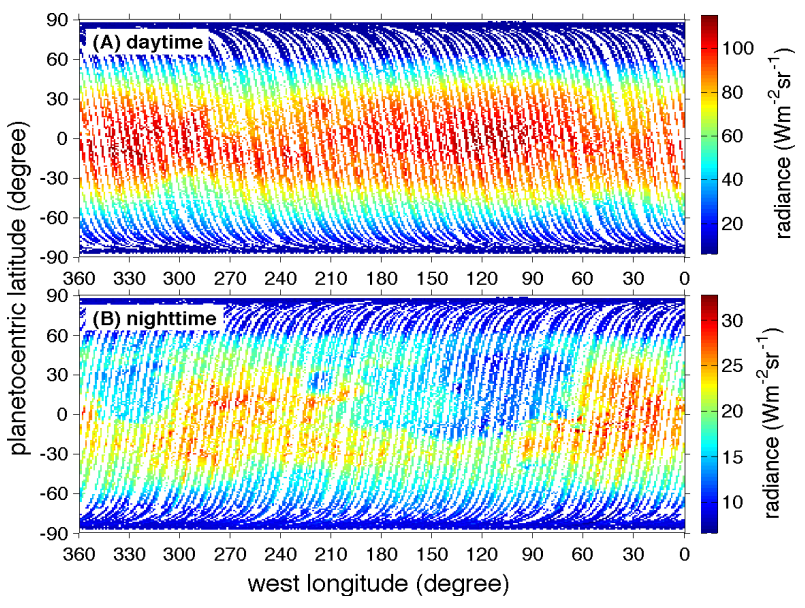

Figure 3. Thermal radiance recorded by the TES (A) Daytime. (B) Nighttime. broadband solar reflectance sensor can be used to compute Mars' Bond albedo and hence the absorbed solar power, but the TES solar reflectance observations have some limitations (e.g., coverage of phase angle). The current available observations from other Mars missions (e.g., Mars Climate Sounder) also have limitations in measuring Mars' global radiant energy budget. Accordingly, better observations (e.g., better coverage of wavelength and view angle) are needed to advance our 
understanding of Mars' global radiant energy budget.

The radiant energy on Mars is tightly coupled to atmospheric and surface properties and processes. Radiant energy measurements should hence be accompanied by measurements of the optical and thermal properties of the atmosphere and surface. The combination of such measurements will lead to a better understanding Mars' global radiant energy budget and the processes controlling it.

Pluto and Triton are similar in many ways. There are very limited studies of the global radiation budgets of the two bodies $(41,42)$. Their Bond albedos are $\sim 0.72 \pm 0.07(43)$ and 0.65 (44) respectively, which are much larger than Mars' albedo 0.37-0.40 (45). Comparative studies of the radiation budgets among these terrestrial bodies with thin atmospheres (e.g., Mars, Pluto, and Triton) will be interesting.

3.1.4 Terrestrial Bodies with thick atmospheres.

In our solar system, Venus is the only terrestrial body with a thick atmosphere. Venus has a strong greenhouse effect (46), and the measurements of radiation budget can help us better determine the efficiency of the greenhouse mechanism. The observations and studies of Venus' radiation budget are very limited (47-50). We do not know how well the absorbed solar energy is balanced by the emitted thermal energy on Venus. The spatial and temporal variations of Venus' radiation budget have not been measured either. Considering the very thick atmosphere on Venus (Venus' surface atmospheric pressure is about 90 times that of Earth) and its highly reflective clouds, we expect that Venus has a unique radiation budget among the terrestrial planets in our solar system.

The observations from the Akatsuki mission can be used to partly address the emitted power of Venus $(50,51)$, but they do not work well for measuring Venus' Bond albedo and hence the global energy budget. Therefore, better observations and studies of the radiation budget of Venus are urgently needed. NASA just selected two Discovery Program Investigations to develop missions to Venus (i.e., VERITAS and DAVINCl+), but the global radiation budget is not the main goal of the two mission concepts. So new missions are still urgently needed for providing the first picture of the global radiant energy budget of Venus.

\subsection{Giant planets}

\subsubsection{Gas Giants}

The emitted power of giant planets is generally thought to be constant with time due to their large thermal inertia. However, Cassini observations suggest that Jupiter's emitted power changes with time (22). Cassini observations also provide the first picture of Jupiter's full-disk albedo in the domain of wavelength and phase angle (18) (see Fig. 4). Based on our measurements of the emitted thermal power and the absorbed solar power, we suggest that Jupiter's Bond albedo and

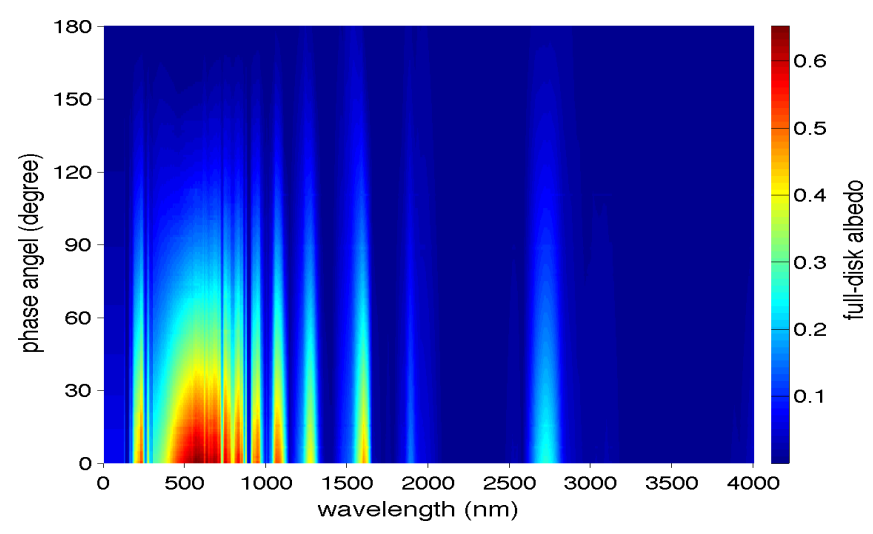

Figure 4. Jupiter's full-disk albedo in the domain of wavelength and phase angle.

internal heat are $0.50 \pm 0.01$ and $7.5 \pm 0.2 \mathrm{~W} / \mathrm{m}^{2}$ respectively $(18)$, which are significantly larger than the previous best estimates $\left(0.34 \pm 0.03\right.$ and $\left.5.4 \pm 0.4 \mathrm{~W} / \mathrm{m}^{2}\right)(53)$. 


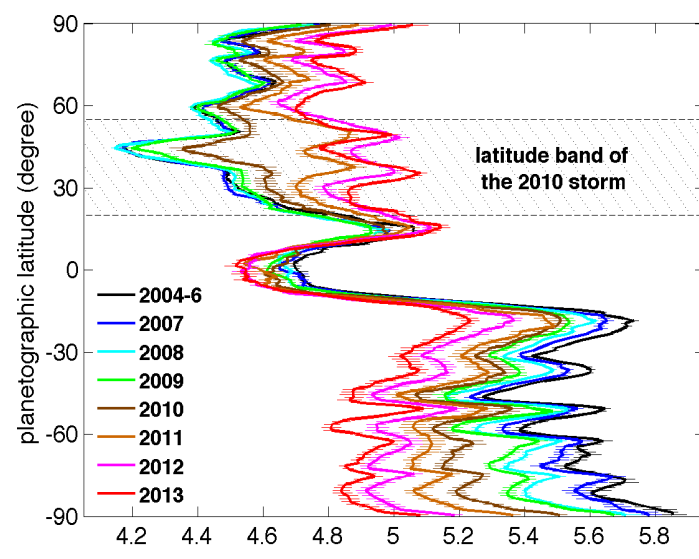

Figure 5. Saturn's emitted power from the Cassini observations.
The Cassini multi-instrument observations are also used to explore Saturn's radiant energy budget and internal heat $(17,23)$. Saturn's emitted power displays seasonal variations (Fig. 5). Such seasonal variations are clearly displayed in the Southern Hemisphere from 2004 to 2013 (23). The variations in the Northern Hemisphere are more complicated, since both seasonal variations and the 2010 giant storm play roles. Measurements of Saturn's absorbed power are more difficult because of effects from the rings, and we are still working to determine the absorbed solar energy and hence the internal heat of Saturn.

The Cassini observations provide a great opportunity to examine the radiant energy budgets and internal heat of Jupiter and Saturn, but there are still limitations. For example, the Cassini observations cannot resolve the temporal variations of Jupiter's radiant energy budget. For Saturn, the Cassini observations provide the first opportunity to examine the temporal variations of its radiant energy budget, but the Cassini observations cover less than half a Saturnian year. Saturn has strong seasonal variations due to its large obliquity and optically thick rings. To better resolve the seasonal cycle of Saturn's radiant energy budget and internal heat, we need observations that cover at least a complete Saturnian year.

\subsubsection{Ice Giants}

The radiant energy budgets and internal heat of Uranus and Neptune have been studied for a long time. But there are discrepancies among these studies of ice giant planets especially for Uranus. Estimates using the ground-based and Voyager observations suggest there is no detectable internal heat $(54,55)$. But detailed modeling $(56)$ suggests that the internal heat of Uranus should be substantially larger than the estimates from observational studies. The dynamo-generated magnetic field also requires an internal heat source (Soderlund et al. white paper, The Underexplored Frontier of Ice Giant Interiors and Dynamos).

The above discrepancy is mainly due to the limitations in previous observational studies. Enough coverage of viewing angle and wavelength is required to compute the radiant energy budget and corresponding internal heat, but significant observational gaps in viewing angle and wavelength exist in the previous observations of Uranus and Neptune $(54,54)$. In addition, there are some issues in the calibration of Voyager visible observations, which were used in these previous estimates. We are re-calibrating these Voyager images (57) and expect better phase functions of fulldisk albedo of Uranus and Neptune.

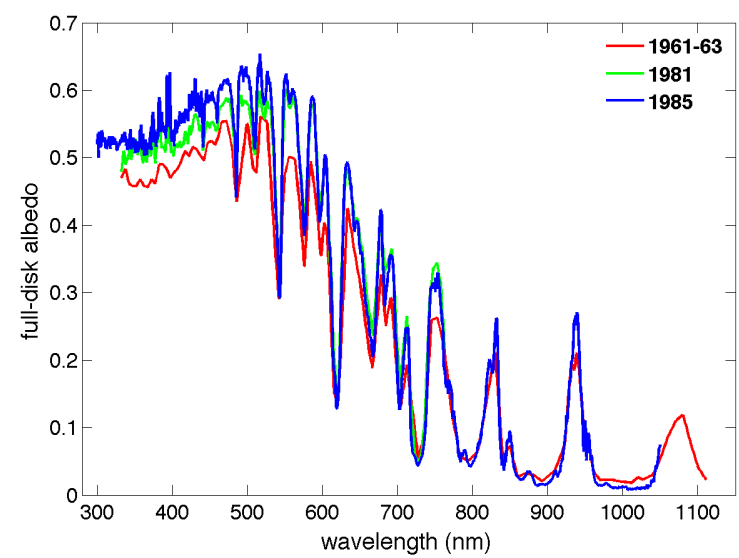

Figure 6. Temporal variations of full-disk albedo of Uranus.

The other limitation in the previous studies is that the temporal variations of the 
radiant energies, which plays important roles in the radiant energy budgets of planets and moons (24), has not been adequately addressed. Figure 6 shows that Uranus' full-disk albedo significantly varied with time especially in the visible wavelengths around $500 \mathrm{~nm}(0.5 \mu \mathrm{m})$ where the solar radiance is concentrated (58-60).

The limitations in the current observations and studies make it difficult to precisely measure the radiant energy budgets and corresponding internal heat of Uranus and Neptune. It is impossible to completely solve the observational limitations by Earth or near-Earth observatories. Therefore, future missions to Uranus and Neptune, which include well-designed instruments measuring their radiant energies (61) and in situ heat fluxes (62), are still needed.

\section{Future Exploration}

In summary, recent studies of radiant energy budgets and internal heat of planets and moons have pointed to energetic processes that are beyond the current planetary science framework. But there are still significant limitations in current studies, which are mainly due to the limited observations (e.g., limited coverage of wavelength and viewing angle) and poor measurement precision. The systematic observations and studies of the global radiant energy budgets for most of the planets and moons in our solar system have not been conducted yet. We think the following science topics have high priorities.

(1) Terrestrial planets. For all terrestrial planets beyond Earth, we do not even know if their global radiant energies are balanced. In particular, the exploration of global radiant budget of Venus should have the highest priority for the following two reasons: (i) As the only terrestrial planet with a dense atmosphere, we expect Venus has a unique energy budget which will help us understand its atmospheric and climate systems; and (ii) The current observations and studies of Venus' global radiant energy budget are very limited.

(2) Giant planets. The seasonal variations of the radiant energy budget play important roles not only in the atmospheric evolution but also the estimate of internal heat of giant planets. Combining the long-term observations from Earth or near-Earth observatories and the precise measurements from the flyby or on-orbit spacecraft can resolve this problem. In particular, investigating the discrepancy among the studies of Uranus' internal heat is urgent because such investigations can help us better develop the evolutionary theories and models of giant planets.

(3) Moons. The exploration of radiant energy budgets of moons can help us better understand their surface features (e.g., ices) and sub-surface internal heat. However, the global radiant energy budgets for most of moons are poorly understood. We thus recommend the studies of the radiant energy budgets of moons based on the current and future observations.

Even with the current observations, it is possible to improve our understanding of radiant energy budgets and internal heat for some planets and moons by better analyses (e.g., combining multi-mission observations to resolve some limitations). But considering the intrinsic limitations in the current observations, new missions and observations are still needed. To significantly advance our understanding of the radiant energy budgets and internal heat of planets and moons, we have the following recommendations.

(1) Support from the decadal survey and NASA. We suggest that the decadal survey supports continued research of the radiant energy budgets and internal heat of planets and moons. Part of these investigations are supported by NASA existing programs (e.g., CDAP and MDAP), but the measurements of the radiant energy budgets and the related critical 
properties and processes are important enough that they should be specifically encouraged in relevant funding AO's.

(2) Instrument/mission development. The current instruments for measuring the radiant energies of planets and moons have some weaknesses (61). So we recommend developing better instruments. The precise measurements of radiant energies require complete coverage of viewing angles and accurate calibration, but such measurements cannot be achieved by Earth and near-Earth observatories. Therefore, on-orbit missions are needed for measuring the radiant energy budgets of planets and moons.

(3) We also recommend the decadal survey to encourage the planetary science community to foster an interdisciplinary, diverse, equitable, inclusive, and accessible environment. It will benefit the exploration of our solar system.

\section{References}

[1] Conrath, B. J. et al. (1989) in Origin and Evolution of Planetary and Satellite Atmospheres. [2] Hanel R. A. et al. (2003) in Exploration of the Solar System by Infrared Remote Sensing. [3] Peixoto J. P. and Oort A. H. (1992) in Physics of Climate. [4] Goody, R. (2007) JAS 64, 2735-2739. [5] Schubert, G. \& Mitchell, J. L. (2013) In Comparative Climatology of Terrestrial Planets. [6] Read, P. L. et al. (2016) QJRMS 142, 703-720. [7] McCleese, D. J. et al. (2007) JGR, doi.org/10.1029/2006JE002790. [8] Smoluchowski, R. (1967) Nature 215, 691-695. [9] Hubbard, W. B. (1968) The Astrophysical Journal 152, 745-754. [10] Salpeter, E. (1973) Astrophys. 181, L83-L86. [11] Verbiscer, A. J. (2006) Icarus 182, 211223. [12] Nimmo, F. et al. (2007) Nature 447, 289. [13] Roberts, J. H. \& Nimmo, F. (2008) GRL, doi.org/10.1029/2008GL033725. [14] Howett, C. J. A. et al. (2011) JGR, doi.org/10.1029/2010JE003718. [15] Spencer, J. R. et al. (2013) EPSC 8, 1-2. [16] Lorenz, E. N. (1955) Tellus 7, 157-167. [17] Phillips, N. A. (1956) QJRMS 82, 123-164. [18] Li, L. et al. (2010) JGR, doi:10.1029/2010JE003631. [19] Li, L. et al. (2018) Nature Communications, doi:10.1038/s41467-018-06107-2. [20] Li, L. (2015) Scientific Reports, doi:10.1038/srep08239. [21] Li, L. et al. (2011) GRL, doi/10.1029/2012EO070017. [22\} Li, L. et al. (2012) $J G R$, doi:10.1029/2012JE00419. [23] Li, L. et al. (2015) GRL, doi:10.1002/2015GL06376. [24] Li, L. et al. (2020) submitted. [25] Creecy, E. et al. (2019) GRL, doi.org/10.1029/2019GL084833. [26] Hansen, J. et al. (2005) Science 308, 1431-1435. [27] Hansen, J. et al. (2011) Atmos. Chem. Phys. 11, 13421-13449. [28] Trenberth, K. E. et al. (2014) J. Climate 27, 3129-3144. [29] Trenberth, K. E. (2016) Journal of Climate 29, 7495-7505. [30] Von Schuckmann, K. et al. (2016) Nature Climate Change 6, 138-144. [31] Tokano, T. et al. (1999) PSS 47, 493-520. [32] Friedson, A. J. et al. (2009). PSS 57, 1931-1949. [33] Newman, C. E. et al. (2011) Icarus 213, 636-654. [34] Lebonnois, S. et al. (2012) Icarus 218, 707- 722. [35] Lora, J. M. et al. (2015) Icarus 250, 516-528. [36] McKay, C. P. et al. (1991) Science 253, 11181121. [37] Paige, D. A. \& Ingersoll, A. P. (1985) Science 228, 1160-1168. [38] Paige, D. A. (1994) JGR 99, 25959-25991. [39] Haberle, R. M. (2013) Icarus 223, 619-620. [40] Christensen, P.R. et al. (1998) Science 279, 1692-1698. [41] Hemelrijck (1982) Icarus 52, 560-564. [42] Brown, R. H. et al. (1991) Science 251,1465-1467. [43] Buratti, B. J. et al. (2017) Icarus 287, 207-217. [44] Nelson, R. M. (1990) GRL 17, 1761-1764. [45] Christensen, P. R. (1988) JGR 93, 7611-7624. [46] Ingersoll, A.P. (1969) JAS 26, 11911198. [47] Tomasko, M. G. et al. (1980) JGR 85, 8187-8199. [48] Titov, D. V. (2007) In GEOPHYSICAL MONOGRAPH-AMERICAN GEOPHYSICAL UNION. [49] Covey, C. et al. (2013) In Comparative Climatology of Terrestrial Planets. [50] Joo Lee et al., (2019) AJ 158, 126-141. [51] Nakamura, M. et al. (2011) Earth, planets and space 63, 443-457. [52] Nakamura, M. et al. (2016) Earth, Planets and Space 68, 1-10. [53] Hanel, R. A. et al. (1981) JGR 86, 8705-8712. [54] Pollack, J. B. et al. (1986) Icarus 65, 442-466. [55] Pearl, J. C. et al. (1990) Icarus 84, 12-28. [56] Marley, M. S. \& McKay, C. P. (1999) Icarus 138, 268-286. [57] Wenkert, D. et al. (2019) AGU, P13B. [58] Younkin, R. (1970) Ph.D. thesis. [59] Lockwood, G. W. et al. (1983) ApJ 266, 402-414. [60] Karkoschka, E. (1998) Icarus 133, 134-146. [61] Li et al. (2019) AGU, P34C. (62) Aslam et al.(2020) Space Sci. Rev. 216, 11. 